\title{
Protective Effect of Vitamin E and Selenium on the Liver, Heart and Aorta
}

\author{
Hossam Fouad Attia ${ }^{1,2^{\star}}$; Mohamed Mohamed Soliman ${ }^{1,3}$ and \\ Tamer Ahmed Ismail ${ }^{1,4}$
}

1 Faculty of Applied Medical Science, Department of Medical laboratories. Taif University, KSA. 2 Histology \& Cytology Department, Faculty of Veterinary Medicine, Benha University, Moshtohor, Egypt, 3 Biochemistry Departmet, Faculty of Veterinary Medicine, Benha University, Moshtohor, Egypt,

4 Physiology Dept. Faculty of Veterinary Medicine, Zagazig University, Egypt.

Received October, accepted for publication December 2011

\section{Abstract}

This study was carried on four groups of Wistar male rats (20 of each), to evaluate the protective effect of vitamin $E$ and selenium on the structure and biochemical alterations of the liver, heart and aorta. Group I is control, Group II fed high fat and vitamin $E$ and selenium deficient diet as control positive group. Group III (protective) given $100 \mathrm{mg}$ and $0.1 \mathrm{mg} / \mathrm{kg}$ b.w/day for 6months, vitamin $\mathrm{E}$ and selenium respectively with high fat diet. Group IV were injected intra peritoneal Vitamin $\mathrm{E}$ and selenium $1 \square \mathrm{g} / 100 \mathrm{~g}$ b.w for 6 months after feeding high fat diet for 6 months. At the end of experimental design, histological section from liver, heart and aorta and so serum changes in metabolites of heart and liver were examined. The tissues of the control posi-

J. Vet. Anat.

tive group showed accumulation of small and large fat globules in the hepatocytes, loss of striation in the cardiac muscles and thickening in the wall of the aorta with loss of the elasticity of the elastic membranes in the tunica media. While the addition of vitamin $E$ and selenium (vitamin $\mathrm{E} 100 \mathrm{mg} . \mathrm{kg} / \mathrm{bw}$ and selenium $0.1 \mathrm{mg} . \mathrm{kg} / \mathrm{b} . \mathrm{w}$ ) to the ration of the third group (protective group), prevent the severe hazard effect that occurred in the second (control positive group). The injections of vitamin $E$ and selenium $1 \mathrm{ug} / 100 \mathrm{~g}$ for 6 months lead to decrease the degenerative changes in the heart, liver and aorta with regeneration of these cells. Vitamin $E$ and selenium administered or injected to Wistar rats decreased significantly the increase in serum levels of LDL, CPK, GOT induced by feeding high fat diet alone. These results collectively 
showed the beneficial effect of vitamin $E$ and selenium as protective antioxidant to cardiac and liver biohazards induced by high fat diet and can be used as protective and therapeutically factor in liver, heart and blood vessels diseases. So, it's preferable to add vitamin $\mathrm{E}$ and selenium to the food in appropriate ratio to avoid the hazard effect of their deficiency, as protective treatment for liver, heart and blood vessels diseases.

\section{Key Words}

Vitamin E and Selenium, Heart, Liver, Protective effects, Wistar rats

\section{Introduction}

Vitamin $E$ is the best-researched fat soluble antioxidant known for its protective effects on lipid membranes and unsaturated fatty acids. Vitamin $E$ is well documented to prevent atherosclerosis and may also help prevent Alzheimer's disease. Its protective effects include the heart, brain, skin, eyes, liver, breasts, and prostate. It stabilizes blood fats so the blood vessels and heart are protected from free-radical induced injury. Selenium benefits treat or prevent some health conditions like, heart diseases, HIV and AIDS, miscarriages, arthritis, muscular degeneration, strokes, gray hair and different types of cancer.
Our body requires a very little amount of selenium. It also promotes antioxidant activity in the body. Selenium has also proved effective in fighting viruses that cause cold sores and shingles. Some studies have shown that consumption of selenium is helpful in making the blood "less sticky" which prevents heart strokes. (Dursun et al, 1998 and Griffith, 2000).

Selenium deficiency in healthy people is relatively rare. However, it can occur in patients with severely compromised intestinal function, or those undergoing total parenteral nutrition. Alternatively, people dependent on food that is sourced from selenium-deficient soil are also at risk. Severe gastrointestinal disorders may decrease the absorption of selenium, resulting in selenium depletion or deficiency. People with selenium deficiency have a greater chance of developing cancer, heart disease, inflammatory disease, cataracts, and signs of premature aging (Schwarz, 1965).

Selenium is an essential trace element for humans and many other forms of life, and a deficiency of this element induces some pathological conditions, such as cancer, coronary heart disease, and liver necrosis (Yoshiro et al., 2003). Selenium is an essential trace mineral that is a component of major antioxidant 
enzymes, selenoproteins (glutathione peroxidase, thioredoxin reductase). Glutathione peroxidase is responsible for detoxification in the body by reducing peroxide free radicals that include lipid peroxide formation in cell membranes. (Stranges et al.,2010)

Vitamin $E$ is necessary for the optimum function and metabolism of the nervous, muscular, circulatory and immune systems, and the latter highlights its importance in maintaining the health (Schwenke, 2002). Its function is basically to prevent the breakdown of oxygen at a cellular level (oxidation) when toxic products including hydrogen peroxide and hydroxyl radicals are produced. These oxidizing agents are powerful tissue poisons (Bendich and Machlin 1988).

Supplementation with vitamin E and selenium had a preventive effect on the elevation of the hepatic, thiobarbituric acid reactive substances (TBARS) and improved the diminished activities of the antioxidative enzymes and the levels of GSH. So the effectiveness of vitamin $\mathrm{E}$ and selenium in reducing hepatic damage (Beytut and Aksakal, 2003). Antioxidative nutrients such as vitamin $E$ and selenium can prevent liver fibrosis induced by carbon tetrachloride (Parola et al., 1992). The aim of the current study is to throw more

light on the protective effect of vitamin $E$ and selenium on the liver, heart and blood vessels (aorta), and to ensure that the prophylaxis is better than treatment by addition of vitamin $E$ and selenium to the food.

\section{Materials and methods}

\section{Materials}

Vitamin $\mathrm{E}$ and selenium was purchased from sigma, Aldrich, LTD Co, USA. Liver and cardiac kits were purchased from Wako pure chemicals, Osaka, Japan and were imported by ADWIA pharmaceutical company, Egypt. Male Wistar rats were bought from Egyptian Co for experimental animals import, Helwan, Egypt.

\section{Animals and experimental design}

Four groups of male Wistar rats, 20 rats per group, were used for this study. Wistar rats were 7 weeks old and of weight 200-250 g. Wistar rats were housed at room temperature (28 \pm 2 0C) with a 12-h light and 12-h dark cycle. Wistar rats were handled daily for 10 days to recover the stress and injection effect. Group I got open access to food intake and water with normal balanced diet as negative control. The $2^{\text {nd }}$ group was exposed to feeding with high fat diet $10 \mathrm{gram} / \mathrm{rat} / \mathrm{day}$ and deficient vitamin $\mathrm{E}$ and selenium for six months as control positive group. The $3^{\text {rd }}$ group (protective 
group) was feed with high fat diet together with vitamin $E$ and selenium $(100 \mathrm{mg}$ and $0.1 \mathrm{mg} / \mathrm{kg} / \mathrm{b} . \mathrm{w} /$ day for six months. The $4^{\text {th }}$ group (treated group) was fed high fat diet and deficient vitamin $E$ and selenium for 6 months then injected with both selenium and Vitamin E 1ug/100 g daily for six months. At the end of the experiment, Wistar rats were killed by decapacitation to get serum and tissues (liver-heart and aorta) for histological and serum analysis.

\section{Histological analysis}

Small pieces of the liver, heart and aorta were kept in Susa fluids, then dehydrated in ascending grades of alcohols, cleared in xylene, embedded in soft paraffin and blocked in hard paraffin. The samples were cut at 5 um thickness and stained with general and special stains according to (Bancroft et al., 1994).

\section{Serum analysis}

Collected blood was left to clot then centrifuged at $8000 \mathrm{rpm}$ at $40 \mathrm{C}$ to get serum. Serum was kept at -20 $\mathrm{OC}$ till assayed. Heart enzymes (LDH and CPK) and Liver parameters (glutamic oxaacetate transaminase [GOT], albumin and uria) were assyed using available spectrophotmetry based kits.

\section{Statistical analysis}

J. Vet. Anat.
Results were expressed as means \pm S.E. of independent experiments. Statistical analysis was done using ANOVA and Fischer's post hoc test, with $p<0.05$ being considered as statistically significant.

\section{Results}

The histology of normal Wistar rats (Figs 1, 2 and 3) showed that the liver consisted of hepatic cords around central vein (Fig 1). The hepatocytes appeared crowded with centrally basophilic nuclei and dark acidophilic cytoplasm. The cardiac muscles appeared long fibers with centrally located multinucleate and acidophilic cytoplasm (Fig 2). The aorta characterized by intact endothelial cells of the tunica intima (tunica interna) and fenestrated elastic lamina of the tunica media (Fig 3) and intact tunica adventitia (tunica externa).

Feeding of Wistar rats high fat and vitamin $E$ and selenium deficient diet induced significant changes in liver, heart and aorta. As seen in Figs (4, 5 and 6), the liver showed foamy cytoplasmic hepatocytes and large centrally located basophilic nuclei (Fig 4). Also, accumulation of different size fat globules in the cytoplasm of the hepatocytes with thickening in the internal elastic lamina of the central vein. The cardiac muscles characterized by loss of 
striation, structureless esosinophilc masses (Fig 5). The aorta showed thickened elastic lamina with loss of elasticity (Fig 6).

When vitamin $\mathrm{E}$ and selenium was given orally to Wistar rats together with high fat diet as a protective therapy, it induced significant changes. As seen in Fig (7), the hepatocytes showed few degenerative changes, as indicated by lymphocytic infiltration around the central vein with congestion in the central vein. The cardiac muscles showed positive PAS reaction and clear muscular striation (Fig 8). The aorta showed few thickened elastic lamina between the normal elastic membranes (Fig 9).

Lastly, when vitamin $E$ and selenium given as therapy, it gave significant changes compared to positive control group. The hepatocytes showed few degenerative changes compared to the control positive group, some hepatocytes showed accumulation of fat globules others showed regeneration in the rest of the cells as seen in Fig 10. The cardiac muscle showed intracardial congestion and hemorrhage, and some cells still showed loss of striation and structureless esosinophilc masses (Fig 11). The aorta showed few regenerative changes as the elastic lamina became zigzag and loss some of its thickened wall (Fig 12).

As seen in table (1). Vitamin E and selenium induced protective effects on heart enzymes (LDH and CPK) relative to Wistar rats given high fat diet alone. As Vitamin E and selenium when used as protective and curative therapy, decreased significantly $(p<0.05)$ the increase in LDH and $C P K$ induced in high fat diet group. Moreover, Vitamin E and selenium is less significant on liver enzymes as Vitamin $E$ and selenium failed to inhibit the high fat diet induced increase in albumin and urea levels. Vitamin $\mathrm{E}$ and selenium shows significant effect on GOT levels as protective factor but not as therapeutic factor.

\section{Discussion}

This study shows that usage of Vitamin $E$ is more beneficial as a protective than as therapeutic vitamin in heart, aorta and liver diseases. Similar findings were reported by Beytut and Aksakal (2001), where the effectiveness of vitamin $E$ and selenium in reducing hepatic damage in glucocorticoid-treated Wistar rats was confirmed. On the same direction, (Naziroğlu and Çay 2000 and Douillet et al., 1998), showed that intraperitoneally administered vitamin $E$ and selenium have significant protective effects on the blood, 
liver, and muscle against oxidative damage of diabetes. As well as Shen et al. (2005) and Aboul-Soud et al. (2011) reported that vitamin $\mathrm{E}$ and selenium supplementation at the given level can inhibit carbon tetra chloride induced activation and proliferation of HSCs ( hepatic stromal cells ) and promote the apoptosis of activated HSCs in acute damage phase. Yanardag et al. (2001) and Mezey et al. (2011), recorded that the combination of selenium and vitamin $\mathrm{E}$, but not either alone protected against hepatic fibrosis by decreasing transforming growth factor- (TGF $\beta 1$ ) - mediated collagen secretion and accumulation by the stellate cells. This protective effect is due to a combination of decreased formation, decreased stability and increased degradation of the collagen. While Yilmaz et al. (1997) recorded that the intra peritoneal administration of DL-alphatocopherol acetate and sodium selenate exert a protective effect against liver radiation damage. Also, Chen and Tappel (1993) found that combination of Vitamin $E$ and selenium and $b$-carotene decreased oxidative damage to rat heart, kidney and lung.

The cardiovascular disease risk is diminished by the vitamin $E$ supplementation. The mechanism of Vitamin $\mathrm{E}$ action is its ability to regulate cell property. As, the cell- regulating properties of vitamin $E$ have been more recently discovered and was taken into account as regards to the functions of platelets, monocytes-macrophages, endothelial cells and vascular smooth muscle cells (Leger 2000, Pryor 2000, Rózewicka etal.,1991 and Yang and Chen 1990). The effect of Vitamin $E$ and selenium on aorta is good and is a promising finding as the addition of vitamin $E$ and selenium leads to improvement in the structure of the aorta. These results were supported by Mehta et al., (2002) and Wójcicki et al. (1991) whom recorded that combination of selenium and vitamin $E$, results in an intensified effect on the improvement of metabolic processes and on the reduction of atherosclerotic plaque formation in rabbits. Parallel to these findings, heart enzymes changes induced by high fat diet were corrected by addition of Vitamin $\mathrm{E}$ and selenium (Table 1).

Vitamin and mineral supplementation have been promoted as a strategy to prevent atherosclerosis. In vitro studies have shown that antioxidants such as vitamins $E$ and $C$, carotene, and selenium reduce lipid peroxidation and free radical damage, which are important intermediaries in the pathogenesis of atherosclerosis Bleys et al., (2006) and Steinberg and Witztum (2002). As, the effects of dietary sodium sele- 
nite and vitamin $\mathrm{E}$ on the microvascular permeability of rat organs such as heart, brain, kidney, liver and eye were investigated by using the Evans blue leakage method. Combined deficiency of selenium and vitamin $E$ caused an increase in the permeability of the heart and eye with respect to their controls while it had no considerable effect on the permeability of other organs. On the other hand, toxic levels of selenium $(4.2 \mathrm{mg} / \mathrm{kg})$ in diet decreased the permeability in kidney, liver, and eye. These results suggested that low or high sodium selenite and vitamin $E$ contents in diet could alter the microvascular permeability of different organs in different manners. It might be important to give reasonable explanations for the pathophysiology of some diseases that are characterized with organ damage and /or dysfunction originated from selenium deficiency or toxicity (Yilmaz et al., 1996).

In conclusion, it is beneficial to use Vitamin $E$ with selenium as factor that can protect liver, heart and aorta from hazard induced by obesity in high fat diet rat. Moreover usage of Vitamin $\mathrm{E}$ and selenium as a protective is better than as a therapeutic vitamin in hepatic and heart diseases.

\section{References}

Aboul-Soud ,A. M.; Abdulaziz M. AlOthman ; Gaber E. ElDesoky ; Zeid A. Al-Othman ; Kareem Yusuf ; Javed Ahmad and Abdulaziz A. AlKhedhairy. (2011): Hepatoprotective effects of vitamin E/selenium against malathion-induced injuries on the antioxidant status and apoptosis-related gene expression in Wistar rats. J. Toxicol. Sci. Vol.36, No.3, 285296, 2011.

Bancroft, J.D; Cook, H.C; Stirling, R.W and Turner,D.R.(1994): " Manual of histological techniques and their diagnostic application' .2nd Ed., Churchill Livingstone, Edinburgh, London, New York.

Bendich, A and Machlin L.J. (1988): Safety of oral intake of vitamin E. Am J Clin Nutr 1988; 48:612-619.

Beytut Ebru and Aksakal Mesut (2003): Effects of dietary vitamin $E$ and selenium on antioxidative defense mechanisms in the liver of Wistar rats treated with high doses of glucocorticoid. Biological trace element research.vol. 91. P: 231-241.

Bleys Joachim; Edgar R Miller III; 
Roberto Pastor-Barriuso; Lawrence J Appel, and Eliseo Guallar (2006): Vitaminmineral supplementation and the progression of atherosclerosis: a meta-analysis of randomized controlled trials: Am J Clin Nutr ; 84: 880.

Chen H and Tappel A L. (1993):

Protection of heme proteins by vitamin $\mathrm{E}$, selenium, and beta-carotene against oxidative damage in rat heart, kidney, lung and spleen. Free Radic Res Commun. ;19 (3): 183-90.

Douillet C; Bost M; Accominotti M;

Borson-Chazot $F$ and Ciavatti M. (1998): Effect of selenium and vitamin $\mathrm{E}$ supplements on tissue lipids, peroxides, and fatty acid distribution in experimental diabetes. Lipids. Apr;33 (4):3939.

Dursun, S.M; Oluboka, O.J ; Deva rajan, $S$ and S.P Kutcher (1998): High-dose vitamin E plus vitamin B6 treatment of risperidone-related neuroleptic malignant syndrome .journal of Psychopharmacology, Vol 12 No 2 P: 220222

Griffith, H. W.(2000): Minerals, Sup plements \& Vitamins: The Essential Guide. New York, NY: Perseus Books Group.
Leger, C. (2000): Vitamin E and cardiovascular prevention. Ann Biol Clin (Paris). SepOct:58(5):527-40.

Mehta U; Kang BP; Kukreja RS and Bansal MP (2002): Ultrastructural examination of rabbit aortic wall following high-fat diet feeding and selenium supplementation: a transmission electron microscopy study. J Appl Toxicol. Nov-Dec; 22(6):405-13.

Mezey E; Liu X and Potter JJ.

(2011): The combination of selenium and vitamin $E$ inhibits type I collagen formation in cultured hepatic stellate cells. Biol Trace Elem Res. Apr;140 (1):82-94. Epub 2010 Mar 25.

Naziroğlu Mustafa and Çay Mehmet (2000): Protective role of intraperitoneally administered vitamin $\mathrm{E}$ and selenium on the antioxidative defense mechanisms in Wistar rats with diabetes induced by streptozotocin. Biol Trace Elem Res. Sep;79 (2):149159.

Parola M; Leonarduzzi G; Biasi F; Albano E; Biocca ME; Poli G and Dianzani MU. (1992): Vitamin E dietary supplementation protects against carbon tetrachloride-induced chronic liver damage and cir- 
rhosis. Hepatology 1992; 16: 1014-1021.

Pryor, W.A. (2000): Vitamin E and heart disease: basic science to clinical intervention trials. Free Radic Biol Med. 2000 Jan 1;28 (1):141-64.

Rózewicka L; Barcew-Wiszniewska B; Wójcicki J; Samochowiec L and Krasowska, B (1991): Protective effect of selenium and vitamin $E$ against changes induced in heart vessels of rabbits fed chronically on a high-fat diet. Kitasato Arch Exp Med. Dec;64(4):183-92.

Schwarz, K. (1965): Role of vitamin $\mathrm{E}$, selenium and related factors in experimental nutritional liver disease. Federation Proc.24: 58.4.

Schwenke, DC. (2002): Does lack of tocopherols and tocotrienols put women at increased risk of breast cancer. J Nutr Biochem. Jan;13(1):2-20 .

Steinberg $D$ and Witztum JL. (2002): Is the oxidative modification hypothesis relevant to human atherosclerosis? Do the antioxidant trials conducted to date refute the hypothesis? Circulation ;105: 2107-11.

Stranges S; Navas-Acien A; Rayman MP and Guallar E. (2010): Selenium status and cardiometabolic health: state of the evidence. Nutr Metab Cardiovasc Dis. Dec;20(10):754-60.

Wójcicki J; Rózewicka L; BarcewWiszniewska B; Samochowiec L, Juźwiak S; Kadłubowska D; Tustanowski S and Juzyszyn Z (1991): Effect of selenium and vitamin $E$ on the development of experimental atherosclerosis in rabbits. Atherosclerosis. Mar; 87 (1): 9-16.

Shen Xiau-Hua ; Wu-Feng Cheng; Xuan-Hai Li ; Jian-Qin Sun; Feng $\mathrm{Li}$, Ling $\mathrm{Ma}$ and LiangMin Xie.(2005): Effects of dietary supplementation with vitamin $E$ and selenium on rat hepatic stellate cell apoptosis. World J Gastroenterol ;11(32): 4957-4961.

Yanardağ R; Bolkent S and Kizir A. (2001): Protective effects of DL-alpha-tocopherol acetate and sodium selenate on the liver of Wistar rats exposed to gamma radiation. Biol Trace Elem Res. Dec; 83 (3): 263-73.

Yang XH and Chen TZ. (1990): Pro tective effect of the antioxidants selenium, vitamin $\mathrm{E}$, and superoxide dismutase on cultured human endothelial cells (ECs) injured by lipid peroxidation. Zhonghua 
Bing Li Xue Za Zhi. Mar; 19 (1): 8-11.

Yilmaz O;Celik S;Cay Mand Naziroğlu M. (1997): Protective role of intraperitoneally administrated vitamin $E$ and selenium on the levels of total lipid, total cholesterol, and fatty acid composition of muscle and liver tissues in Wistar rats. J Cell Biochem.Feb;64(2):23341.

Yilmaz,Emine Demirel; Deniz

Dinçer; Gürsel Yilmazand Belma Turam (1996): The effect of selenium and vitamin
$E$ on microvascular permeability of rat organs. Biological trace Element Research. Vol. 64. P: 161-175.

Yoshiro Saito;Yasukazu Yoshida;

Takashi Akazawa; Kazuhiko

Takahashi and Etsuo Niki.

'; ,2003): Cell Death Caused by Selenium Deficiency and Protective Effect of Antioxidants. J. of biological chemistry.Vol. 278 , No. 41 , Issue of October 10, pp. 3942839434.

Correspondence to: Hossam Fouad Attia, Department of Histology \& Cytology, Faculty of Veterinary Medicine, Benha University, Egypt.

Drhossam222@hotmail.com

\begin{tabular}{|c|c|c|c|c|}
\hline & Normal diet & HFD & $\begin{array}{c}\text { HFD+Vit.E\&Se } \\
\text { food }\end{array}$ & $\begin{array}{c}\text { HFD+Vit.E\&Se } \\
\text { injection }\end{array}$ \\
\hline LDH & $1442.5 \pm 19.67$ & $\begin{array}{c}4751.25 \pm \\
132.8^{*}\end{array}$ & $2826.33 \pm 92.9 \#$ & $3144 \pm 74.5 \#$ \\
\hline CPK & $144.33 \pm 4.8$ & $684 \pm 33.86^{*}$ & $442 \pm 12.4 \#$ & $382 \pm 5.6 \#$ \\
\hline Albumin & $4 \pm 0.07$ & $4.95 \pm 0.32$ & $3.3 \pm 0.33^{*}$ & $4.53 \pm 0.145$ \\
\hline GOT & $149.75 \pm 14.8$ & $342.15 \pm 48.04 \%$ & $310.66 \pm 66.4 *$ & $273.33 \pm 10.13 \#$ \\
\hline Urea & $24.75 \pm 1.9$ & $22.75 \pm 1.3$ & $53.66 \pm 11.2 *$ & $29.66 \pm 1.45^{*}$ \\
\hline
\end{tabular}

Table (1): Cardiac and liver function test in Wistar rats. Wistar rats were exposed to high fat diet (HFD) alone or together with Vitamin $E$ in food or injection. As seen Vitamin $E$ induced protective effects on heart enzymes ( $\mathrm{LDH}$ and $\mathrm{CPK}$ ) relative to Wistar rats given fat diet alone. Vitamin $E$ is less significant on liver functions as Vitamin $E$ failed to inhibit the diet induced increase in albumin, GOT and urea levels. ${ }^{*} p<0.05$ relative to normal Wistar rats. $\# p<0.05$ relative to high fat diet Wistar rats. 

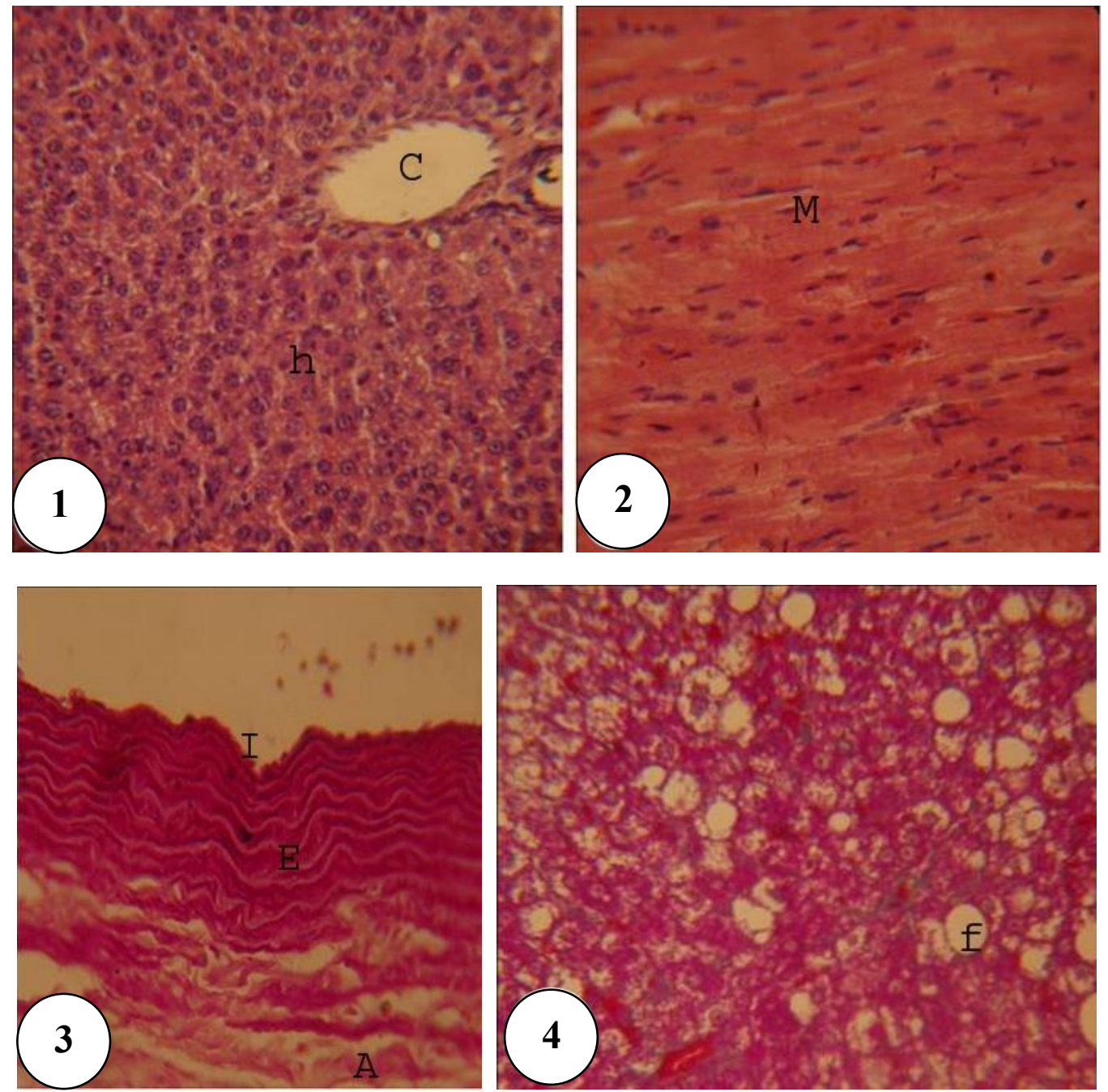

Fig (1): Photomicrograph of the male Wistar rats liver of the control group showing; central vein (C) and hepatic cords (h). H\&E, X10

Fig (2): Photomicrograph of the male Wistar rats heart of the control group showing cardiac muscle (M) H\&E, X10

Fig (3): Photomicrograph of the male Wistar rats aorta of the control group showing; tunica intima (tunica interna) (I) tunica media (E) and tunica adventitia (tunica externa) (A ).PAS, X10

Fig (4): Photomicrograph of the male Wistar rats liver of the control positive group showing; accumulation of small and large size fat globules that replaced the hepatocytes $(\mathrm{F})$.Crossmon's trichrome, $\mathrm{X} 20$ 

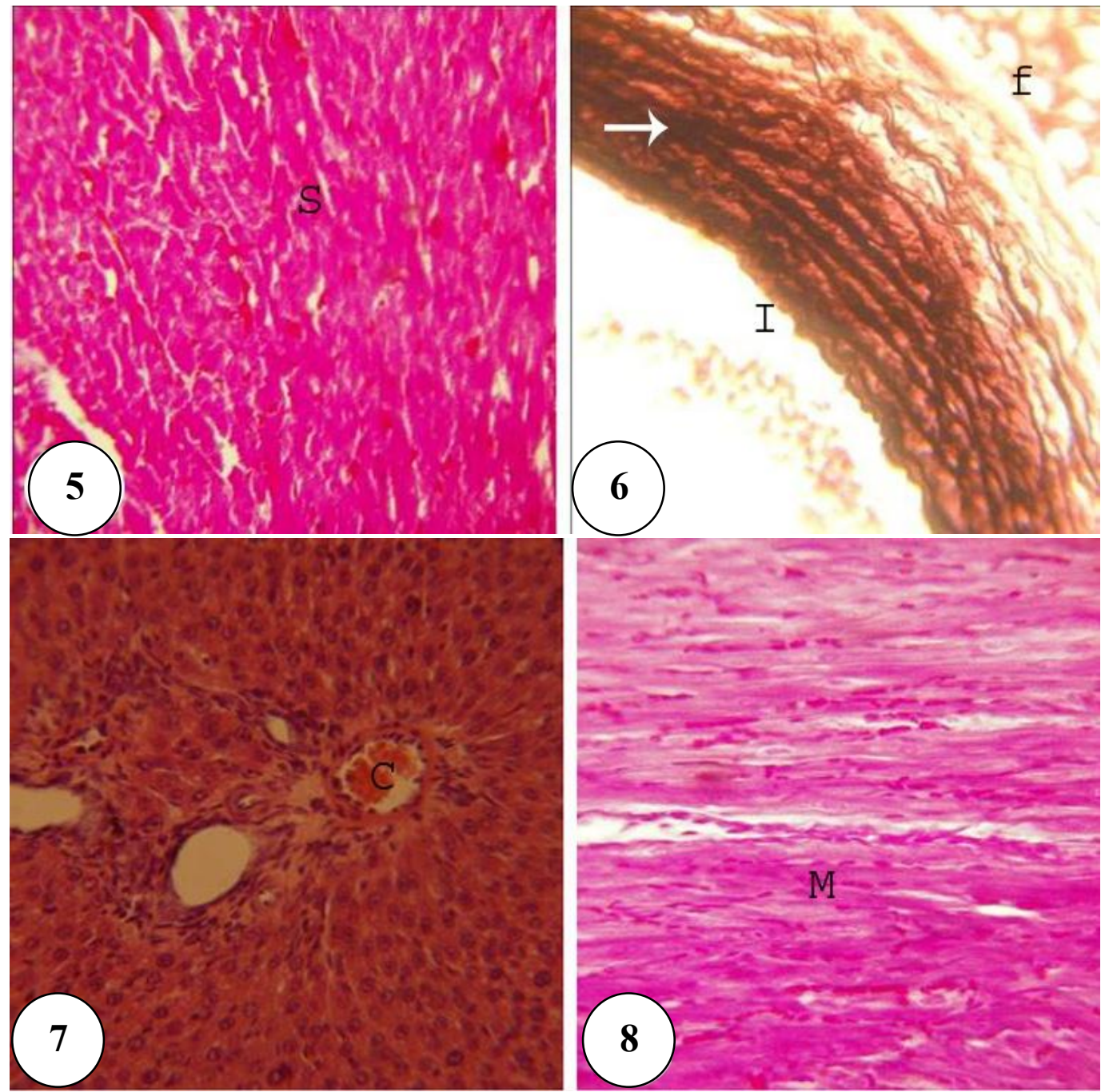

Fig (5): Photomicrograph of the male Wistar rats heart of the control positive group showing eosinophilic structureless masses (S) Crossmon's trichrome, X40

Fig (6): Photomicrograph of the male Wistar rats aorta of the control positive group showing; tunica intima (tunica interna) (I) thick elastic membranes of the tunica media (arrow) and accumulation of adipose tissues around the aorta (f). Weighert elastic tissue stain, X20

Fig (7): Photomicrograph of the male Wistar rats liver of the protective group showing; regeneration of the hepatocytes, congestion of the blood vessels $(C) H \& E, X 20$

Fig (8): Photomicrograph of the male Wistar rats heart of the protective group showing, regeneration of the cardiac muscle (M). Crossmon's trichrome, X20 

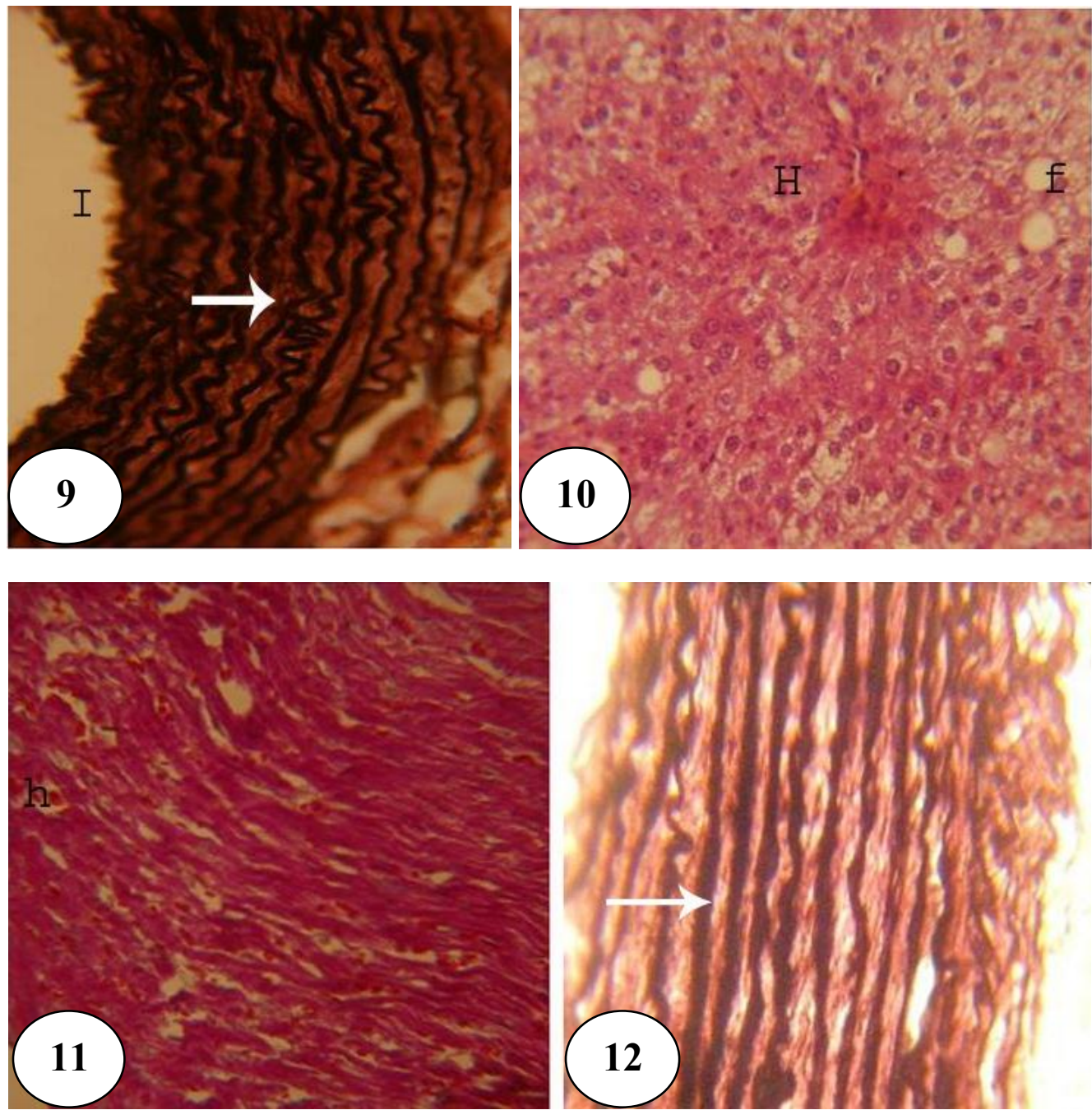

Fig (9): Photomicrograph of the male Wistar rats aorta of the protective group showing; tunica intima (tunica interna) (I) and fenestrated elastic membranes (arrow) .Weighert elastic tissue, $\mathrm{X} 40$

Fig (10): Photomicrograph of the male Wistar rats liver of the treated group showing; regeneration of the hepatocytes $(\mathrm{H})$ and some fat globules still replaced the hepatocytes (F). H\&E, X20

Fig (11): Photomicrograph of the male Wistar rats heart of the treated group showing , regeneration of the cardiac muscle, and intracardial congestion and hemorrhage $(H)$ Crossmon's trichrome, X40

Fig (12): Photomicrograph of the male Wistar rats aorta of the treated group showing; thick elastic membranes (arrow) .Weighert elastic tissue stain, X40 